\title{
Sazonalidade e qualidade do sêmen fresco e criopreservado de touros Pantaneiro e Nelore*
}

\section{Seasonality and quality of fresh and cryopreserved semen of Pantaneiro and Nellore bulls}

\author{
Luis Eduardo Senra e Silva, ${ }^{* *, * * *}$ Luciana Keiko Hatamoto-Zervoudakis, ${ }^{* *, * * *}$ Alexandre Floriani Ramos, ${ }^{* * * *}$ \\ Joanis Tilemahos Zervoudakis, ${ }^{* * *}$ Pedro Paulo Tsuneda, ${ }^{* *, * * *}$ Moacir Ferreira Duarte Júnior, ${ }^{* *, * * *}$
}

Fabiana Mariani Wingert, ${ }^{* * * * *}$ Juliana de Oliveira Moraes ${ }^{* *}$

\begin{abstract}
Resumo
Objetivou-se avaliar o efeito da estação do ano sobre a qualidade do sêmen fresco e criopreservado de reprodutores Pantaneiros (Bos taurus) criados em condições tropicais. Foram utilizados 7 touros Pantaneiros e 3 Nelores (controle), dos quais foi aferido circunferência escrotal, consistência testicular e após a coleta e congelamento do sêmen realizada análise de motilidade, vigor, defeitos menores, maiores e totais, concentração, integridade de membrana plasmática e acrossomal para sêmen fresco e além destas, estresse oxidativo para sêmen criopreservado. O experimento foi conduzido em delineamento inteiramente casualizado, e arranjo fatorial $2 \times 2$ ( 2 raças e 2 estações do ano). A raça, estação do ano ou a interação entre eles, não alteraram significativamente as médias de circunferência escrotal, consistência testicular, motilidade, vigor, concentração, integridade de membrana acrossomal e porcentagem de defeitos menores. A integridade da membrana plasmática no sêmen fresco sofreu efeito da estação do ano e foi menor no inverno em ambas raças $(95,76 \pm 1,77 \%$ vs. $87,07 \pm 4,78 \% \mathrm{P}=0,03)$. A estação do inverno aumentou a porcentagem de defeitos maiores $(29,15 \%$ vs. $16,44 \%, P<0,01)$ e totais $(17,49 \%$ vs. $30,45 \%, P<0,01)$. Os parâmetros do sêmen congelado não foram influenciados pela raça, estação do ano ou interação entre elas. Portanto, nas condições edafoclimáticas estudadas, os reprodutores Pantaneiros apresentaram redução na sua qualidade seminal na estação do inverno.
\end{abstract}

Palavras-chave: estresse oxidativo, temperatura ambiente, raças localmente adaptadas.

\begin{abstract}
The aim of the present study was to evaluate the effect of seasonality on the quality of fresh and cryopreserved semen of Pantaneiro breed (Bos taurus) bulls raised under tropical conditions. Scrotal circumference and testicular consistency were performed in seven Pantaneiro and three Nellore (control) bulls. Sperm motility, vigor, minor, major and total defects, concentration, plasma and acrosomal membrane integrity were assessed in fresh and post-thawed semen. Additionally, oxidative stress was determined in post-thawed semen samples. The experiment was done in a complete randomized design, with a $2 \times 2$ factorial arrangement $(2$ breeds and 2 seasons). Breed, season of the year or the interaction of both did not alter scrotal circumference, testicular consistency, motility, vigor, concentration, acrosomal membrane integrity and percentage of minor defects $(P<0.05)$. Plasma membrane integrity of fresh semen was affected by the season and was smaller in winter in both breeds $(95.76 \pm 1.77 \%$ vs $87.07 \pm 4.78 \%, P=0.03)$. Nevertheless, also in the winter there was an increase in the percentage of major $(29.15 \%$ vs $16.44 \%, P<0.01)$ and total defects $(17.49 \%$ vs. $30.45 \%, P<0.01)$ in fresh semen samples. Breed, season or interaction of both did not influence the sperm parameters of cryopreserved semen. Thus, in the studied climatic conditions, the Pantaneiro breed bulls present decrease in semen quality mainly in the winter season.
\end{abstract}

Keywords: oxidative stress, environment temperature, locally adapted breeds.

\section{Introdução}

O bovino Pantaneiro (Bos taurus) tem em sua origem as raças ibéricas, que chegaram ao Brasil através de seus colonizadores (Portugal e Espanha) e passaram por seleção natural em ambientes extremos, como o Pantanal (Mazza et al., 1992). Hoje é considerada uma raça localmente adaptada, pois suportam condições que podem ser inóspitas para outras raças como: áreas alagadas e oferta de forragem nativa de baixa disponibilidade e qualidade (Mariante et al., 2009). Desta maneira esta raça pode contribuir significativamente para a pecuária, especialmente em regiões onde há a necessidade

\footnotetext{
${ }^{*}$ Recebido em 24 de agosto de 2016 e aceito em 23 de abril de 2017.

**Laboratório de Biotecnologia e Reprodução Animal, Universidade Federal de Mato Grosso, Avenida Fernando Corrêa da Costa, 2367, Boa Esperança, 78060-900, Cuiabá-MT, Brasil.

***Programa de Pós-Graduação em Ciência Animal, Universidade Federal de Mato Grosso.

****Embrapa Recursos Genéticos e Biotecnologia, Brasília-DF, Brasil.
} 
de características relacionadas à rusticidade e adaptabilidade (Egito et al., 2002).

A partir da década de 1930, houve uma forte introdução na região do Pantanal principalmente da raça Nelore, o que provocou diminuição do contingente do bovino Pantaneiro, embora haja alguns núcleos de conservação in situ, a raça corre risco de ser extinta (Mariante e Egito, 2002). A criopreservação de sêmen é uma das melhores maneiras de conservar este recurso genético e disseminá-lo com o uso de biotecnologias (Mariante et al., 2011).

Animais submetido a altas temperaturas têm queda na fertilidade pois o seu parênquima testicular entra em um quadro de hipóxia e consequente degeneração, que por sua vez leva a maiores níveis de estresse oxidativo no ejaculado, aumento de defeitos espermáticos, queda na motilidade, entre outros (Nichi et al., 2006; Magalhães Jr et al., 2016).

A fim de conhecer o efeito da sazonalidade sobre os parâmetros seminais dos bovinos Pantaneiros, objetivou-se avaliar a qualidade do sêmen fresco e congelado de touros Pantaneiros (Bos taurus) durante o verão e inverno bem como verificar se, em relação aos parâmetros reprodutivos, estes animais estão adaptados às condições edafoclimáticas do pantanal sul-matogrossense.

\section{Material e métodos}

\section{Animais e local do experimento}

Foram utilizados 7 reprodutores da raça Pantaneiro (Bos taurus) e 3 da raça Nelore (Bos indicus) com idade média de 48 meses e peso corporal médio de $606,1 \pm 92,09 \mathrm{Kg}$, mantidos em pasto de Brachiaria brizantha cv. Marandu, com suplemento proteicoenergético nas águas, proteico na seca e água ad libitum. Os animais eram provenientes de uma fazenda no município de Rochedo-MS, que está situado a $260 \mathrm{~m}$ acima do nível do mar. O clima da região é tropical, caracterizado por verão chuvoso e inverno seco. A precipitação pluviométrica anual varia entre 1500 a $1750 \mathrm{~mm}$.

As coletas e congelamentos do sêmen foram realizados a campo nos meses de janeiro e junho de 2013 (sendo uma coleta por animal em cada época do ano) e a análise das amostras foi realizada nos laboratórios de Biotecnologia e Reprodução Animal (LABRA) e Biologia Molecular da Universidade Federal de Mato Grosso, campus Cuiabá.

\section{Coleta, congelamento e avaliações seminais}

Circunferência escrotal foi mensurada através de fita métrica e consistência testicular (1-5, onde 1-flácido e 5-rígido) por meio de palpação (Unaniam et al., 2000). O sêmen foi obtido pelo método de eletroejaculação e coletado em tubo graduado de $15 \mathrm{~mL}$. Foi realizado as análises imediatas: motilidade (MOT) e vigor (VIG) espermáticos sob microscopia óptica em aumento de 400x (CBRA, 2013), uma alíquota foi diluída em formol salino na proporção de 1:200 para análise de concentração (CONC) que foi realizada em câmara de Neubauer (CBRA, 2013) e morfologia espermática em microscopia de contraste de fase (Barth \& Oko, 1989), foram contadas 200 células e classificadas em defeitos maiores (DEFMA) e menores (DEFME) (Blom, 1973). Além disso, foi feito o preparo de lâminas de eosina-nigrosina para a avaliação da integridade de membrana plasmática (WHO, 1992) e coloração de Pope para integridade da membrana acrossomal (IMA) (POPE et al., 1991).

Após avaliação imediata, cada ejaculado foi diluído em extensor tris-gema $(3,187 \mathrm{~g}$ TRIS-hidroximetil amino metano; $1,78 \mathrm{~g}$ ácido cítrico monohidratado; $1,316 \mathrm{~g}$ frutose; $80 \mathrm{~mL}$ água destilada; $20 \mathrm{~mL}$ gema de ovo; $100 \mathrm{mg}$ de estreptomicina; $100000 U \mathrm{U}$ de penicilina; $5 \mathrm{~mL}$ de glicerol para cada $100 \mathrm{~mL}$ de diluidor) (Rota et al., 1995) em fração única na concentração de $40 \times 10^{6}$ espermatozoides $/ \mathrm{mL}$, envasado em palhetas de 0,5 $\mathrm{mL}$ e submetido à seguinte curva de congelamento: 4 horas de resfriamento $\left(4^{\circ} \mathrm{C}\right) ; 15$ minutos em contato com o vapor de nitrogênio (a $5 \mathrm{~cm}$ do nitrogênio líquido, $-120^{\circ} \mathrm{C}$ ); em seguida submergidas em nitrogênio líquido e por fim armazenadas em botijão criogênico $\left(-196^{\circ}\right)$.

As amostras foram descongeladas a $37 \mathrm{C}^{\circ}$ durante 30 segundos e $10 \mu \mathrm{L}$ foram utilizados para análise de motilidade e vigor espermáticos em microscopia óptica sob o aumento de 400x (CBRA, 2013). Foram avaliados ainda integridade de membrana plasmática e acrossomal em microscopia óptica sob o aumento de 1000x, com 200 células contadas em ambas análises.

O estresse oxidativo espontâneo foi calculado através da quantificação das substâncias reativas ao ácido tiobarbitúrico (TBARS) segundo a metodologia de Buege e Aust (1978). Após a descongelação, a amostra de sêmen foi lavada com a finalidade de remover os resquícios de diluidor, com a adição de $1600 \mu \mathrm{L}$ de PBS, centrifugada a $(800 \times \mathrm{g}, 10 \mathrm{~min})$ e descartado o sobrenadante. O pellet de $500 \mu \mathrm{L}$ de sêmen foi misturado a $1000 \mu \mathrm{L}$ de uma solução a $10 \%$ de ácido tricloroacético gelado (TCA 10\%), homogeneizados e centrifugados (18,000 × g, 15 min, $15^{\circ} \mathrm{C}$ ) para a precipitação de proteínas. Após centrifugação uma alíquota do sobrenadante foi misturado com $500 \mu \mathrm{L}$ de ácido tiobarbitúrico a $1 \%$ (TBA $1 \%$, diluído em $0,05 \mathrm{~N}$ de $\mathrm{NaOH}$ ) em tubos e incubado em água fervente $\left(90\right.$ a $\left.100^{\circ} \mathrm{C}\right)$ por $15 \mathrm{~min}$. Após este período, os tubos contendo a mistura foram imediatamente resfriados em banho de gelo $\left(0^{\circ} \mathrm{C}\right)$ para parar a reação (Ohkawa et al., 1979). A absorbância das amostras foi então quantificada utilizando-se um espectrofotômetro em comprimento de onda de $532 \mathrm{~nm}$. Os resultados foram comparados a uma curva padrão previamente preparada com solução de MDA (malondialdeído) em quantidades conhecidas e crescentes. A concentração de TBARS foi determinada utilizando um valor de $1.56 \times 10^{5} \mathrm{M} /$ $\mathrm{mL}$ com o coeficiente de extinção molar do MDA. $O$ índice de peroxidação lipídica foi descrito em nanogramas de TBARS por $10^{6}$ espermatozoides.

\section{Análise estatística}

O experimento foi realizado em delineamento inteiramente casualizado, em arranjo fatorial $2 \times 2$ (2 raças e 2 estações) e as médias foram analisados através da ANOVA e comparados pelo teste de média de Tukey com nível de significância de 5\%, o programa estatístico utilizado foi o SAS (versão 9.2).

\section{Resultados e discussão}

A raça, estação do ano ou a interação entre eles, não alteraram significativamente as médias de circunferência escrotal, consistência testicular, motilidade, vigor, concentração, integridade de membrana acrossomal e porcentagem de defeitos menores. Contudo a integridade da membrana plasmática sofreu influência da estação do ano e foi menor no inverno (Tabela 1). 
No presente estudo, a circunferência escrotal e consistência testicular não sofreram alteração em função da estação do ano, no gado Pantaneiro, possivelmente pela adaptação da raça as condições edafoclimáticas encontradas na região central do Brasil, já que esta foi introduzida na região há aproximadamente 300 anos (Mazza et al., 1992). De acordo com Johnson et al. (2000) essa diminuição do parênquima testicular pode estar relacionada ao aumento da temperatura do ambiente e consequentemente do testículo. Contudo as temperaturas no local onde foi conduzido o estudo se mantiveram estáveis ao longo do ano e não foram suficientes para provocar alteração nas características testiculares.

Já Teixeira et al. (2011) encontraram diminuição do comprimento e volume testicular de touros Curraleiros (raça localmente adaptada) criados no centro oeste brasileiro nos meses de abril e maio em comparação aos outros meses do ano, segundo os autores porque estão meses são caracterizados pelo início da estação seca.

Em nosso experimento foi encontrado aumento da porcentagem de defeitos maiores e consequentemente totais $(P<0,01)$ no inverno em comparação ao verão (Tabela 1). Contrariamente, Nichi et al. (2006), encontraram maior porcentagem de defeitos maiores no verão, no ejaculado de touros Simental (Bos taurus) em comparação aos Nelore (Bos indicus) criados em condições tropicais no centro oeste brasileiro.

No presente trabalho tanto reprodutores Pantaneiros (Bos taurus), quanto os animais da raça Nelore (Bos indicus) não apresentaram queda na qualidade seminal durante o verão. Frequentemente têm sido relatada na literatura maior resistência ao calor pelos bovinos de origem indiana e maior suscetibilidade pelos europeus (Nichi et al., 2006; Magalhães Jr et al., 2016). Contudo, os Pantaneiros provavelmente não apresentaram queda na qualidade seminal porque se tornaram mais resistentes ao estresse térmico, devido à sua adaptação.

As temperaturas registradas na região onde foi conduzido o experimento foram semelhantes durante todo o ano, sendo que a umidade diminuiu significativamente no inverno. Nesse sentido, o aumento da porcentagem de defeitos maiores no inverno pode estar relacionado com a baixa disponibilidade e qualidade das forragens na época seca do ano, uma vez que, alguns estudos têm revelado queda na qualidade seminal quando os animais passam por restrição alimentar (Barth et al., 2008).

Segundo Barth et al. (2008), indicadores metabólicos como leptina, insulina, GH e IGF-1 sinalizam ao hipotálamo que reduza os pulsos de $\mathrm{GnRH}$ quando em situações de balanço energético negativo. Além disso, ingestão insuficiente de proteína não fornece substrato suficiente para a produção de hormônios proteicos envolvidos na produção de espermatozoides como $\mathrm{LH}$ e FSH.

A motilidade, vigor, concentração de TBARS e integridade de membrana acrossomal e plasmática (Tabela 2) após o descongelamento não foram afetadas pela raça, estação do ano ou a interação entre eles.

Tabela 1: Médias e valores de $P$ de variáveis analisadas em sêmen fresco de acordo com a raça (Nelore x Pantaneiro), estação (verão x inverno) e a interação entre eles

\begin{tabular}{|c|c|c|c|c|c|c|c|}
\hline \multirow{2}{*}{ Variáveis $^{a}$} & \multicolumn{2}{|c|}{ Verão } & \multicolumn{2}{|c|}{ Inverno } & \multicolumn{3}{|c|}{ Probabilidade $(P)$} \\
\hline & Nelore & Pantaneiro & Nelore & Pantaneiro & $\mathrm{R}^{\mathrm{b}}$ & $\mathrm{E}^{\mathrm{c}}$ & $\mathrm{R}^{\mathrm{b}} \times \mathrm{E}^{\mathrm{c}}$ \\
\hline CE & $38,33 \pm 0,88$ & $38,07 \pm 1,04$ & $37,33 \pm 1,30$ & $36,78 \pm 1,50$ & 0,7942 & 0,4648 & 0,9266 \\
\hline CONSIST & $3,00 \pm 0,00$ & $3,00 \pm 0,00$ & $3,33 \pm 0,33$ & $2,85 \pm 0,14$ & 0,1334 & 0,536 & 0,1334 \\
\hline MOT & $75,00 \pm 7,63$ & $70,71 \pm 3,99$ & $76,66 \pm 4,40$ & $65,83 \pm 4,90$ & 0,1894 & 0,7741 & 0,5605 \\
\hline VIG & $3,00 \pm 0,00$ & $2,85 \pm 0,14$ & $3,33 \pm 0,33$ & $3,08 \pm 0,20$ & 0,3663 & 0,2044 & 0,8029 \\
\hline CONC & $545 \pm 142$ & $572 \pm 448$ & $497 \pm 119$ & $488 \pm 872$ & 0,7463 & 0,2575 & 0,6125 \\
\hline IMP & $94,16 \pm 2,45$ & $96,44 \pm 1,10$ & $89,50 \pm 5,77$ & $85,62 \pm 3,80$ & 0,8078 & 0,0308 & 0,3558 \\
\hline IMA & $92,86 \pm 2,15$ & $97,31 \pm 1,13$ & $92,00 \pm 4,33$ & $92,84 \pm 2,34$ & 0,2903 & 0,2875 & 0,4668 \\
\hline DEFME & $2,16 \pm 1,09$ & $0,57 \pm 0,31$ & $1,25 \pm 0,25$ & $1,33 \pm 0,16$ & 0,1542 & 0,8797 & 0,1166 \\
\hline DEFMA & $12,5 \pm 5,5$ & $18,14 \pm 3,53$ & $36,5 \pm 2,5$ & $26,00 \pm 3,83$ & 0,6256 & 0,0056 & 0,1195 \\
\hline DEFTO & $14,66 \pm 6,05$ & $18,71 \pm 3,71$ & $37,75 \pm 2,25$ & $27,33 \pm 3,91$ & 0,5418 & 0,0076 & 0,1774 \\
\hline TBARS & $90,24 \pm 16,17$ & $120,22 \pm 16,32$ & $121,32 \pm 26,91$ & $178,3 \pm 20,22$ & 0,3934 & 0,3424 & 0,6442 \\
\hline
\end{tabular}

${ }^{a} \mathrm{CE}$, circunferência escrotal; CONSIST, consistência testicular; MOT, motilidade; VIG, vigor; CONC, concentração; IMP, integridade de membrana plasmática; IMA, integridade de membrana acrossomal; DEFME, defeitos menores; DEFMA, defeitos maiores; DEFTO, defeitos totais; TBARS, substâncias reativas ao ácido tiobarbitúrico. ${ }^{\mathrm{b} R a c ̧ a ; ~ ' E s t a c ̧ a ̃ o ~ d o ~ A n o . ~}$

Tabela 2: Médias e desvio padrão dos valores de motilidade (MOT), vigor (VIG), integridade de membrana plasmática (IMP) e integridade de membrana acrossomal (IMA) do sêmen descongelado e valor de $\mathrm{P}$ para raça (Nelore $\mathrm{x}$ Pantaneiro) estação (verão x inverno) e a interação entre eles

\begin{tabular}{|c|c|c|c|c|c|c|c|}
\hline \multirow{2}{*}{ Variáveis $^{a}$} & \multicolumn{2}{|c|}{ Verão } & \multicolumn{2}{|c|}{ Inverno } & \multicolumn{3}{|c|}{ Valor de P } \\
\hline & Nelore & Pantaneiro & Nelore & Pantaneiro & $\mathrm{R}^{\mathrm{b}}$ & $\mathrm{E}^{\mathrm{c}}$ & $R \times E$ \\
\hline MOT (\%) & $26,66 \pm 1,66$ & $34,28 \pm 3,99$ & $28,33 \pm 1,66$ & $32,00 \pm 7,00$ & 0,5332 & 0,7489 & 0,2867 \\
\hline VIG (0-5) & $1,33 \pm 0,33$ & $2,14 \pm 0,14$ & $2,00 \pm 0$ & $2,00 \pm 0,31$ & 0,1306 & 0,3164 & 0,1306 \\
\hline IMP (\%) & $21,81 \pm 10,87$ & $27,78 \pm 3,18$ & $24,75 \pm 6,49$ & $20,23 \pm 7,96$ & 0,9223 & 0,757 & 0,4847 \\
\hline IMA (\%) & $94,66 \pm 1,16$ & $97,42 \pm 0,66$ & $97,25+0,72$ & $96,33 \pm 0,76$ & 0,3098 & 0,4099 & 0,0535 \\
\hline TBARS (ng/ml) & $90,24 \pm 16,17$ & $120,22 \pm 16,32$ & $121,32 \pm 26,91$ & $178,3 \pm 20,22$ & 0,3934 & 0,3424 & 0,6442 \\
\hline
\end{tabular}

aMOT, motilidade; VIG, vigor; IMP, integridade de membrana plasmática, IMA, integridade de membrana acrossomal; TBARS, substâncias reativas ao ácido tiobarbitúrico; ${ }^{\circ}$ Raça; 'Estação do ano; médias seguidas de letras minúsculas diferentes na mesma linha, diferem estatisticamente entre si $(P<0,05)$. 
Neste experimento, foi encontrado maior proporção de espermatozoides com defeitos maiores no inverno, porém não foi possível observar aumento da lipoperoxidação. Embora muitas vezes o estresse oxidativo seja correlacionado com a presença de defeitos espermáticos, isso porque espermatozoides com patologias têm maior capacidade de geração de EROs (Oliveira et al., 2006). Além disso, neste trabalho foi mensurado a concentração de TBARS no sêmen descongelado e diluído o que pode ter provocado também diluição da EROs.

Em relação à quantidade de substâncias reativas ao ácido tiobarbitúrico (TBARS), frequentemente trabalhos têm comprovado seu aumento no ejaculado de reprodutores bovinos em situações de altas temperaturas, sendo as raças de origem europeia mais sensíveis (Silva et al., 2009; Nichi et al., 2006).

\section{Referências}

BARTH, A.D.; OKO, R. Abnormal morphology of bovine spermatozoa. Ames: lowa State University Press; 1989.

BARTH, A.D.; BRITO, L.F.C.; KASTELIC, J.P. The effect of nutrition on sexual development of bulls. Theriogenology. v. 70, p. 485-494, 2008.

BLOM, E. The ultrastructure of some characteristic sperm defects and a proposal for a new classification of the bull spermogram. Nordisk Veterinaermedicin. v. 25, p. 383-391, 1973.

BRITO, L.F.C.; SILVA, A.E.D.F.; BARBOSA, R.T.; KASTELIC, J.P. Testicular thermoregulation in Bos indicus crossbred and Bos taurus: relationship with scrotal, testicular vascular cone and testicular morphology, and effects on semen quality and sperm production. Theriogenology. v. 61, p. 511-528, 2004.

BUEGE, J.A.; AUST, S.D. Microsomal lipid peroxidation. Methods of Enzymology. v. 52, p. 302-310, 1978.

COLÉGIO BRASILEIRO DE REPRODUÇÃO ANIMAL-CBRA. Manual para exame andrológico e avaliação de sêmen animal. 3. ed. Belo Horizonte, 104 p, 2013.

EGITO, A.A.; MARIANTE, A.S.; ALBUQUERQUE, M.S.M. The Brazilian Genetic Resources Conservation Programm. Archivos de Zootecnia. v. 51, p. 39-52, 2002.

MAGALHÃES JR, M.J.; MARTINS, L.F.; SENRA, R.L.; SANTOS, T.F.; OKANO, D.S.; PEREIRA, P.R.G.; FARIA-CAMPOS, A. Differential abundances of four forms of Binder of SPerm 1 in the seminal plasma of Bos taurus indicus bulls with different patterns of semen freezability. Theriogenology. v. 83, p. 766-777, 2016.

MARIANTE, A.S.; EGITO, A.A. Animal genetic resources in Brazil: result of five centuries of natural selection. Theriogenology. v. 57, p. 223-235, 2002

MARIANTE, A.S.; ALBUQUERQUE, M.S.M.; EGITO, A.A.; McMANUS, C.; LOPES, M.A.; PAIVA, S.R. Present status of the conservation of livestock genetic resources in Brazil. Livestock Science. v. 120, p. 204-212, 2009.

MARIANTE, A.S.; ALBUQUERQUE, M.S.M.; RAMOS, A.F. Criopreservação de recursos genéticos animais brasileiros. Revista Brasileira de Reprodução Animal. v. 35, p. 64-68, 2011.
Isto se deve ao fato de que os Bos indicus são mais eficientes em realizar a termorregulação, (Brito et al., 2004), no entanto, apesar de ser um Bos taurus a raça Pantaneiro parece ter aumentado sua capacidade de troca de calor o que implica em menores níveis de substâncias reativas ao ácido tiobarbitúrico (TBARS).

\section{Conclusões}

Os reprodutores Pantaneiros (Bos taurus) apresentam redução na sua qualidade seminal no inverno nas condições tropicais encontradas no presente estudo. No que diz respeito à criopreservação seminal, embora não tenha sido encontrado efeito da estação do ano, mais estudos são necessários para a obtenção de melhores resultados.

MAZZA, M.C.M.; MAZZA, C.A.; SERENO, J.R.B.; SANTOS, S.A.L.; MARIANTE, A.S. Conservation of Pantaneiro cattle in Brazil. Historical origin. Archivos Zootecnia. v. 41, p. 443-453, 1992.

NICHI, M.; BOLS, P.E.J.; ZÜGE, R.M.; BARNABE, V.H.; GOOVAERTS, I.G.F.; BARNABE, R.C.; CORTADA, C.N.M. Seasonal variation in semen quality in Bos indicus and Bos taurus bulls raised under tropical conditions. Theriogenology. v. 66, p. 822-828, 2006.

OHKAWA, H.; OHISHI, N.; YAGI, K. Assay for lipid peroxides in animal tissues by thiobarbituric acid reaction. Analytical Biochemistry, v. 95, p. 351-358, 1979.

OLIVEIRA, K.M.; DUARTE, A.M.; NASCIMENTO, M.R.B.M.; DINIZ, E.G.; TAVARES, M.; VIEIRA, R.C. Influência das estações seca e chuvosa sobre as características seminais de touros das raças Nelore, Gir e Holandês criados à pasto. Veterinária Notícias. v. 12, p. 145-151, 2006.

POPE, C.E.; ZHANG, Y.Z.; DRESSER, B.L. A simple staining method for evaluating acrossomal status of cat spermatozoa. Journal of Zoo and Wildlife Medicine. v. 22, p. 87-95, 1991.

ROTA, A.; STROM, B.; LINDE-FORSEBERG, C. Effects of seminal plasma and three extenders on canine semen stored at $4^{\circ} \mathrm{C}$. Theriogenology. v. 44, p. 885-900, 1995.

SILVA, A.R.; FERRAUDO, A.S.; PERECIN, D.; LIMA, V.F.M.H. Efeito da idade do touro e do período da colheita de sêmen de bovinos de raças europeias e zebuínas. Revista Brasileira de Zootecnia, v. 38, p. 1218-1222, 2009.

TEIXEIRA, H.C.A.; NASCIMENTO, N.V.; McMANUS, C.; EGITO, A.A.; MARIANTE, A.S.; RAMOS, A.F. Seasonal influence on semen traits and freezability from locally adapted Curraleiro bulls. Animal Reproduction Science, v. 125, p. 56-61, 2011.

UNANIAM, M.M.; SILVA, A.M.D.F.; McMANUS, C.; CARDOSO, E.P. Características biométricas testiculares para avaliação de touros zebuínos da raça Nelore. Revista Brasileira de Zootecnia. v. 29 , p. $136-144,2000$.

WHO World Health Organization. Who laboratory manual for the examination of human semen and semen - cervical mucus interaction. Cambridge: The Press Syndicate of the University of Cambridge. p. 120, 1992. 